\title{
CREEPING FLOW PAST A POROUS APPROXIMATELY SPHERICAL SHELL: STRESS JUMP BOUNDARY CONDITION
}

\author{
D. SRINIVASACHARYA ${ }^{\otimes 1}$ and M. KRISHNA PRASAD ${ }^{1}$
}

(Received 4 March, 2011; revised 22 June, 2011)

\begin{abstract}
The creeping flow of an incompressible viscous liquid past a porous approximately spherical shell is considered. The flow in the free fluid region outside the shell and in the cavity region of the shell is governed by the Navier-Stokes equations. The flow within the porous annular region of the shell is governed by Brinkman's model. The boundary conditions used at the interface are continuity of the velocity, continuity of the pressure and Ochoa-Tapia and Whitaker's stress jump condition. An exact solution for the problem and an expression for the drag on the porous approximately spherical shell are obtained. The drag is evaluated numerically for several values of the parameters governing the flow.
\end{abstract}

2010 Mathematics subject classification: primary 76A05; secondary 76D07.

Keywords and phrases: porous approximately spherical shell, Stokes flow, Brinkman equation, stress jump coefficient.

\section{Introduction}

Several researchers have studied the flows of fluids past porous particles, as they are of great importance in industrial and engineering applications. These applications include flow through porous beds (fixed or fluidized), sedimentation of fine particulate suspensions, modelling of polymer macromolecule coils in a solvent, catalytic reactions where porous pellets are used, floc settling processes and the flow of oil in oil fields or reservoirs during oil recovery. Problems of flow past porous particles have been modelled by using Stokes' version of the Navier-Stokes equations for the flow outside the porous particles and Darcy's law or Brinkman's equation for the flow within the porous particles. Joseph and Tao [8] considered the creeping flow past a porous spherical shell immersed in a uniform viscous incompressible fluid, using Darcy's law for the flow inside the porous region and Stokes' equations for the fluid

\footnotetext{
${ }^{1}$ Department of Mathematics, National Institute of Technology, Warangal - 506 004, A.P., India; e-mail: dsc@nitw.ac.in, dsrinivasacharya@yahoo.com; kpm973.nitw@gmail.com. (C) Australian Mathematical Society 2011, Serial-fee code 1446-1811/2011 \$16.00
} 
outside the sphere. They took the boundary conditions as continuity of normal velocity and pressure at the surface of the porous sphere and no slip of the tangential velocity component of the free fluid, and found that the drag on the porous sphere is the same as that on a rigid sphere with smaller radius. Beavers and Joseph [1] noted, in connection with experimental investigations of viscous flow past planar permeable surfaces, that a slip occurs at the boundary, and proposed a slip boundary condition. Using a statistical approach to extend Darcy's law to nonhomogeneous porous media, Saffman [17] gave a theoretical justification of the condition proposed by Beavers and Joseph in the limit of small permeability. Neale et al. [12] proved that the condition of Saffman was the most satisfactory. Therefore, for small permeability, Saffman's condition is more appropriate than the usual no-slip condition. Jones [7] solved the problem of the slow viscous flow past a porous spherical shell using a generalization of the Beavers and Joseph [1] boundary conditions for curved surfaces. Using the same boundary conditions, Srinivasacharya [18] considered the flow of an incompressible viscous fluid past and within a porous approximately spherical shell.

Darcy's law appears, however, to be inadequate for flows with high porosities and large shear rates, and for flows near the surface of the bounded porous medium. To model such flows, a modification of Darcy's law was proposed by Brinkman [4] and Debye and Bueche [5] independently. The validity of their equation was confirmed by the experimental verification of Ooms et al. [15] and Matsumoto and Suganuma [11]. Qin and Kaloni [16] considered the creeping flow past and through a porous sphere using Brinkman's law for the flow inside the porous region and Stokes' equations for the fluid outside the sphere. Zlatanovski [19] studied the axisymmetric flow past a porous prolate spheroidal particle using the Brinkman model for the flow inside the spheroidal particle and Stokes' model for the free flow region. Bhatt and Sacheti [2] studied the flow past a porous spherical shell using the Brinkman model. All of these authors $[2,16,19]$ used continuity of the velocity, pressure and tangential stresses as boundary conditions. Recently, by applying volume average techniques, OchoaTapia and Whitaker $[13,14]$ investigated the boundary conditions at the porous-liquid interface and showed that the equations require a discontinuity in the shearing stress but continuity in velocity components and normal stress. They derived the stress jump boundary condition

$$
\epsilon^{-1} \frac{\partial u^{p}}{\partial y}-\frac{\partial u^{l}}{\partial y}=\frac{\sigma}{\sqrt{k}} u^{p},
$$

where $u^{p}$ and $u^{l}$ are tangential velocity components in the porous region and the liquid region, respectively, $\epsilon$ is the porosity, $k$ is the permeability of the homogeneous portion of the porous region and $\sigma$ is the stress jump coefficient. If $\sigma \neq 0$, there is a discontinuity in the shear stress at the porous-liquid interface. This jump condition is constructed to join Darcy's law with the Brinkman correction to Stokes' equations. It has been verified experimentally that $\sigma$ varies in the range -1 to $1[13,14]$. Kuznetsov $[9,10]$ used this stress jump boundary condition at the interface between a porous medium and a clear fluid to discuss flow in channels partially filled with 
a porous medium. Bhattacharyya and Raja Sekhar [3] used a stress jump boundary condition while investigating the Stokes flow of a viscous fluid inside a sphere with internal singularities, enclosed by a porous spherical shell, and found the dependence of the drag and torque on the stress jump coefficient.

In this paper, we consider the creeping flow past a porous approximately spherical shell using a slip condition at the interface proposed by Ochoa-Tapia and Whitaker [13, 14]. The flow examined is axially symmetric in nature. The flow equations are based on the Stokesian version of the Navier-Stokes equations in the general viscous flow regime and the use of Brinkman's model in the porous region. The drag experienced by the porous approximately spherical shell is evaluated numerically with varying permeability for different values of the stress jump coefficient.

\section{Formulation of the problem}

Consider the creeping flow of an incompressible Newtonian viscous fluid past and within a porous approximately spherical shell of average external and internal radii $a$ and $b$ (with $a>b)$. Let $(r, \theta, \phi)$ denote a spherical polar coordinate system with unit basis vectors $\left(\vec{e}_{r}, \vec{e}_{\theta}, \vec{e}_{\phi}\right)$. Assume that there is a uniform velocity $U$ far away from the shell along the axis of symmetry $\theta=0$. Let the equation of the outer approximate sphere be $r=a\left[1+\sum_{m=2}^{\infty} \beta_{m} \vartheta_{m}(\zeta)\right]=: r_{a}$ and that of the inner approximate sphere be $r=b\left[1+\sum_{m=2}^{\infty} \gamma_{m} \vartheta_{m}(\zeta)\right]=: r_{b}$, where the $\beta_{m}$ and $\gamma_{m}$ are small, $\zeta=\cos \theta$ and $\vartheta_{n}(\zeta)$ is the Gegenbauer function [6] of the first kind of order $n$ and degree $-1 / 2$. If all of the $\beta_{m}$ and $\gamma_{m}$ are zero, the approximately spherical shell reduces to a spherical shell of external and internal radii $a$ and $b$. The external region $\left(r \geq r_{a}\right)$, porous region $\left(r_{b} \leq r \leq r_{a}\right)$ and cavity region $\left(r \leq r_{b}\right)$ are denoted as regions I, II and III, respectively. These assumptions are illustrated in Figure 1.

Assume that the flow in both regions I and III, namely inside and outside of the approximately spherical shell, is governed by Stokes' approximation to the NavierStokes equations,

$$
\begin{gathered}
\nabla \cdot \vec{q}^{(i)}=0, \\
\nabla p^{(i)}+\mu \nabla \times \nabla \times \vec{q}^{(i)}=0, \quad i=1,3,
\end{gathered}
$$

and that the flow in region II is governed by Brinkman's model,

$$
\begin{gathered}
\nabla \cdot \vec{q}^{(2)}=0, \\
\nabla p^{(2)}+\frac{\mu}{k} \vec{q}^{(2)}+\mu \nabla \times \nabla \times \vec{q}^{(2)}=0,
\end{gathered}
$$

where $\vec{q}^{(i)}$ is the fluid velocity, $\mu$ is the coefficient of viscosity, $p^{(i)}$ is the pressure and $k$ is the permeability of the porous medium. The superscripts $i=1,2$ and 3 correspond to the fluid properties in the regions I, II and III, respectively.

Since the flow is in the meridian plane and is axially symmetric, all the physical quantities are independent of $\phi$. Hence, we assume that

$$
\vec{q}^{(i)}=u^{(i)}(r, \theta) \vec{e}_{r}+v^{(i)}(r, \theta) \vec{e}_{\theta}, \quad i=1,2,3 .
$$




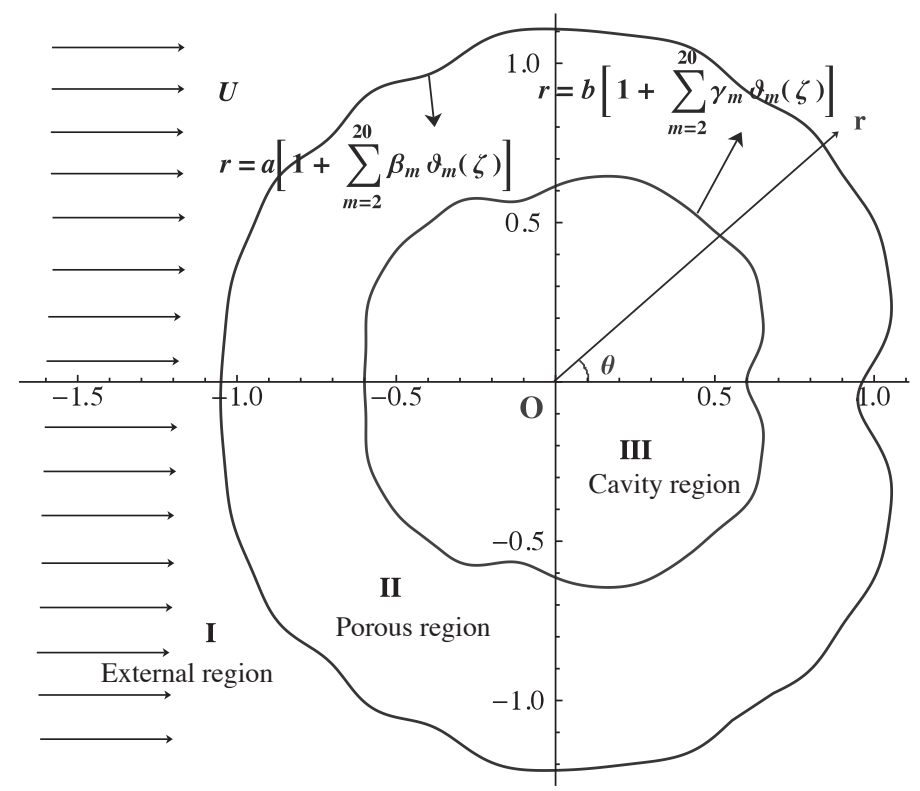

FIGURE 1 . The physical situation and the coordinate system $(m \leq 20, a=1, b=0.6)$.

In view of the incompressibility condition $\nabla \cdot \vec{q}^{(i)}=0, i=1,2,3$, we introduce the stream functions $\psi^{(i)}(r, \theta)$ through

$$
u^{(i)}=-\frac{1}{r^{2} \sin \theta} \frac{\partial \psi^{(i)}}{\partial \theta}, \quad v^{(i)}=\frac{1}{r \sin \theta} \frac{\partial \psi^{(i)}}{\partial r}, \quad i=1,2,3 .
$$

Eliminating pressure from (2.1) and (2.2), and substituting (2.3) into the resulting equations, we obtain the following dimensionless equations for $\psi^{(i)}$ :

$$
\begin{gathered}
E^{4} \psi^{(1)}=0, \\
E^{2}\left(E^{2}-\alpha^{2}\right) \psi^{(2)}=0, \\
E^{4} \psi^{(3)}=0,
\end{gathered}
$$

where $\alpha^{2}=a^{2} / k$ and

$$
E^{2}=\frac{\partial^{2}}{\partial r^{2}}+\frac{\left(1-\zeta^{2}\right)}{r^{2}} \frac{\partial^{2}}{\partial \zeta^{2}}
$$

is the Stokesian stream function operator.

\section{Boundary conditions}

To determine the flow velocity and pressure outside and within the porous annular approximately spherical region and within the cavity region, we use the following boundary conditions to link the different flow regimes. 
(i) Continuity of velocity components on the boundary of the approximate shell:

$$
\begin{array}{llll}
u^{(1)}(r, \theta)=u^{(2)}(r, \theta) & \text { and } & v^{(1)}(r, \theta)=v^{(2)}(r, \theta) & \text { on } r=r_{a}, \\
u^{(2)}(r, \theta)=u^{(3)}(r, \theta) & \text { and } & v^{(2)}(r, \theta)=v^{(3)}(r, \theta) & \text { on } r=r_{b}
\end{array}
$$

(ii) Continuity of pressure on the boundary:

$$
\begin{array}{ll}
p^{(1)}(r, \theta)=p^{(2)}(r, \theta) & \text { on } r=r_{a}, \\
p^{(2)}(r, \theta)=p^{(3)}(r, \theta) & \text { on } r=r_{b} .
\end{array}
$$

(iii) Ochoa-Tapia and Whitaker's $[13,14]$ stress jump boundary condition for tangential stresses, where $\sigma$ is the stress jump coefficient:

$$
\begin{array}{ll}
\frac{\partial v^{(2)}}{\partial r}-\frac{\partial v^{(1)}}{\partial r}=\frac{\sigma}{\sqrt{k}} v^{(2)} & \text { on } r=r_{a}, \\
\frac{\partial v^{(2)}}{\partial r}-\frac{\partial v^{(3)}}{\partial r}=\frac{\sigma}{\sqrt{k}} v^{(2)} & \text { on } r=r_{b} .
\end{array}
$$

Additionally, we have the regularity conditions at infinity,

$$
\lim _{r \rightarrow \infty} u^{(1)}(r, \theta)=-U \cos \theta, \quad \lim _{r \rightarrow \infty} v^{(1)}(r, \theta)=U \sin \theta,
$$

and the condition that velocity and pressure must be nonsingular everywhere.

\section{Solution of the problem}

For region I, the solution of (2.4) which is regular at infinity, that is, far away from the shell and on the axis, is found using separation of variables to be

$$
\psi^{(1)}=\left[r^{2}+\frac{A_{2}}{r}+B_{2} r\right] \vartheta_{2}(\zeta)+\sum_{n=3}^{\infty}\left[A_{n} r^{-n+1}+B_{n} r^{-n+3}\right] \vartheta_{n}(\zeta)
$$

For region II, the solution of (2.5) is

$$
\begin{aligned}
\psi^{(2)}= & {\left[C_{2} r^{2}+\frac{D_{2}}{r}+E_{2} \sqrt{r} K_{3 / 2}(\alpha r)+F_{2} \sqrt{r} I_{3 / 2}(\alpha r)\right] \vartheta_{2}(\zeta) } \\
& +\sum_{n=3}^{\infty}\left[C_{n} r^{n}+D_{n} r^{-n+1}+E_{n} \sqrt{r} K_{n-1 / 2}(\alpha r)+F_{n} \sqrt{r} I_{n-1 / 2}(\alpha r)\right] \vartheta_{n}(\zeta)
\end{aligned}
$$

and for region III, the solution of (2.6) is

$$
\psi^{(3)}=\left[G_{2} r^{2}+H_{2} r^{4}\right] \vartheta_{2}(\zeta)+\sum_{n=3}^{\infty}\left[G_{n} r^{n}+H_{n} r^{n+2}\right] \vartheta_{n}(\zeta)
$$

where $I_{n-1 / 2}(\alpha r)$ and $K_{n-1 / 2}(\alpha r)$ denote the modified Bessel functions of the first kind and second kind (respectively) of order $n-1 / 2$ and $\vartheta_{n}(\zeta)$ is the Gegenbauer function 
of the first kind of order $n$ and degree $-1 / 2$. Here $A_{n}, B_{n}, C_{n}, D_{n}, E_{n}, F_{n}, G_{n}$ and $H_{n}$ for $n \geq 2$ are constants to be determined.

Using (4.1) in (2.1) and integrating the resulting equation, we obtain the pressure $p^{(1)}$ outside the body:

$$
p^{(1)}=-\frac{B_{2}}{r^{2}} P_{1}(\zeta)+\sum_{n=3}^{\infty} B_{n}\left(\frac{6-4 n}{n}\right) r^{-n} P_{n-1}(\zeta) .
$$

Similarly, using (4.2) in (2.2) and (4.3) in (2.1) and integrating, we find the pressure $p^{(2)}$ within the porous annular region and $p^{(3)}$ within the cavity region:

$$
\begin{aligned}
& p^{(2)}=\alpha^{2}\left[C_{2} r-\frac{D_{2}}{2 r^{2}}\right] P_{1}(\zeta)+\alpha^{2} \sum_{n=3}^{\infty}\left[C_{n} \frac{r^{n-1}}{n-1}-D_{n} \frac{r^{-n}}{n}\right] P_{n-1}(\zeta), \\
& p^{(3)}=-10 H_{2} r P_{1}(\zeta)-\sum_{n=3}^{\infty} H_{n}\left(\frac{4 n+2}{n-1}\right) r^{n-1} P_{n-1}(\zeta) .
\end{aligned}
$$

\section{Determination of arbitrary constants}

In dimensionless form, in terms of the stream functions, the boundary conditions from (3.1)-(3.3) are

$$
\begin{gathered}
\psi^{(1)}(r, \theta)=\psi^{(2)}(r, \theta), \quad \psi_{r}^{(1)}(r, \theta)=\psi_{r}^{(2)}(r, \theta), \quad \psi_{r r}^{(2)}-\psi_{r r}^{(1)}=\alpha \sigma \psi_{r}^{(2)}, \\
p^{(1)}(r, \theta)=p^{(2)}(r, \theta) \quad \text { on } r=1+\sum_{m=2}^{\infty} \beta_{m} \vartheta_{m}(\zeta) ; \\
\psi^{(2)}(r, \theta)=\psi^{(3)}(r, \theta), \quad \psi_{r}^{(2)}(r, \theta)=\psi_{r}^{(3)}(r, \theta), \quad \psi_{r r}^{(2)}-\psi_{r r}^{(3)}=\alpha \sigma \psi_{r}^{(2)}, \\
p^{(2)}(r, \theta)=p^{(3)}(r, \theta) \quad \text { on } r=\eta\left[1+\sum_{m=2}^{\infty} \gamma_{m} \vartheta_{m}(\zeta)\right],
\end{gathered}
$$

where $\eta=b / a$.

We first develop the solution corresponding to the boundaries $r=1+\beta_{m} \vartheta_{m}(\zeta)$ and $r=\eta\left[1+\gamma_{m} \vartheta_{m}(\zeta)\right]$. Assume that the coefficients $\beta_{m}$ and $\gamma_{m}$ are sufficiently small so that squares and higher powers of $\beta_{m}$ and $\gamma_{m}$ can be neglected [6]. Comparison of (4.1)-(4.3) with the equations obtained in the case of the flow of an incompressible viscous fluid past a porous spherical shell indicates that the terms involving $A_{n}, B_{n}, C_{n}$, $D_{n}, E_{n}, F_{n}, G_{n}$ and $H_{n}$ for $n>2$ are extra terms which are not present in the case of a spherical shell. The body that we are considering is an approximately spherical shell, and the flow generated is not expected to be much different to that corresponding to the spherical shell [2]. Also, the coefficients $A_{n}, B_{n}, C_{n}, D_{n}, E_{n}$ and $F_{n}$ for $n>2$ are of order $\beta_{m}$, and $C_{n}, D_{n}, E_{n}, F_{n}, G_{n}$ and $H_{n}$ for $n>2$ are of order $\gamma_{m}$. Therefore, while implementing the boundary conditions, we ignore the departure from the spherical form and set, in (5.1), $r=1$ in the terms involving $A_{n}, B_{n}, C_{n}, D_{n}, E_{n}$ and $F_{n}$ and, in (5.2), $r=\eta$ in the terms involving $C_{n}, D_{n}, E_{n}, F_{n}, G_{n}$ and $H_{n}$ (both for $n>2$ ). 
Using the observations made above and the boundary conditions (5.1) and (5.2) in the expressions (4.1)-(4.3), and equating leading coefficients to zero in the resulting equations, we obtain a system of equations in $A_{2}, B_{2}, C_{2}, D_{2}, E_{2}, F_{2}, G_{2}$ and $H_{2}$. Using identities given by Happel and Brenner [6, p. 142], we obtain $A_{n}=B_{n}=C_{n}=$ $D_{n}=E_{n}=F_{n}=G_{n}=H_{n}=0$ for $n \neq m-2, m, m+2$ and another system of equations in $A_{n}, B_{n}, C_{n}, D_{n}, E_{n}, F_{n}, G_{n}$ and $H_{n}$ for $n=m-2, m, m+2$. Solving these two systems of equations gives expressions for the arbitrary constants $A_{n}, B_{n}, C_{n}, D_{n}, E_{n}$, $F_{n}, G_{n}$ and $H_{n}$ for $n=2, m-2, m, m+2$. As the expressions for these constants are lengthy, we do not present them here.

In the case where the approximately spherical shell is defined by the boundaries

$$
r=a\left[1+\sum_{m=2}^{\infty} \beta_{m} \vartheta_{m}(\zeta)\right] \text { and } r=b\left[1+\sum_{m=2}^{\infty} \gamma_{m} \vartheta_{m}(\zeta)\right]
$$

we employ the above technique for each $m$ and obtain expressions for the stream functions for the regions I, II and III by superposition:

$$
\begin{aligned}
\psi^{(1)}=\left[r^{2}\right. & \left.+\frac{A_{2}}{r}+B_{2} r\right] \vartheta_{2}(\zeta)+\sum_{m=2}^{\infty}\left\{\left[A_{m-2} r^{-m+3}+B_{m-2} r^{-m+5}\right] \vartheta_{m-2}(\zeta)\right. \\
& \left.+\left[A_{m} r^{-m+1}+B_{m} r^{-m+3}\right] \vartheta_{m}(\zeta)+\left[A_{m+2} r^{-m-1}+B_{m+2} r^{-m+1}\right] \vartheta_{m+2}(\zeta)\right\}, \\
\psi^{(2)}=[ & \left.C_{2} r^{2}+\frac{D_{2}}{r}+E_{2} \sqrt{r} K_{3 / 2}(\alpha r)+F_{2} \sqrt{r} I_{3 / 2}(\alpha r)\right] \vartheta_{2}(\zeta) \\
& +\sum_{m=2}^{\infty}\left\{\left[C_{m-2} r^{m-2}+D_{m-2} r^{-m+3}+E_{m-2} \sqrt{r} K_{m-5 / 2}(\alpha r)\right.\right. \\
& \left.+F_{m-2} \sqrt{r} I_{m-5 / 2}(\alpha r)\right] \vartheta_{m-2}(\zeta)+\left[C_{m} r^{m}+D_{m} r^{-m+1}+E_{m} \sqrt{r} K_{m-1 / 2}(\alpha r)\right. \\
& \left.+F_{m} \sqrt{r} I_{m-1 / 2}(\alpha r)\right] \vartheta_{m}(\zeta)+\left[C_{m+2} r^{m+2}+D_{m+2} r^{-m-1}+E_{m+2} \sqrt{r} K_{m+3 / 2}(\alpha r)\right. \\
& \left.\left.+F_{m+2} \sqrt{r} I_{m+3 / 2}(\alpha r)\right] \vartheta_{m+2}(\zeta)\right\}, \\
\psi^{(3)}=\left[G_{2}\right. & \left.r^{2}+H_{2} r^{4}\right] \vartheta_{2}(\zeta)+\sum_{m=2}^{\infty}\left\{\left[G_{m-2} r^{m-2}+H_{m-2} r^{m}\right] \vartheta_{m-2}(\zeta)\right. \\
& \left.+\left[G_{m} r^{m}+H_{m} r^{m+2}\right] \vartheta_{m}(\zeta)+\left[G_{m+2} r^{m+2}+H_{m+2} r^{m+4}\right] \vartheta_{m+2}(\zeta)\right\} .
\end{aligned}
$$

\section{Drag on the shell}

The drag force acting on the porous approximately spherical shell is

$$
D=2 \pi a^{2} \int_{0}^{\pi}\left[\tau_{r r}^{(1)} \cos \theta-\tau_{r \theta}^{(1)} \sin \theta\right]_{r=a\left[1+\sum_{m=2}^{\infty} \beta_{m} \vartheta_{m}(\zeta)\right]} \sin \theta d \theta .
$$

Expanding the integrand in terms of the stream function $\psi^{(1)}$, this can be written as

$$
D=2 \pi a^{2} \int_{0}^{\pi} r^{3} \sin ^{3} \theta \frac{\partial}{\partial r}\left(\frac{1}{r^{2} \sin ^{2} \theta} E^{2} \psi^{(1)}\right) r d \theta .
$$


(a)

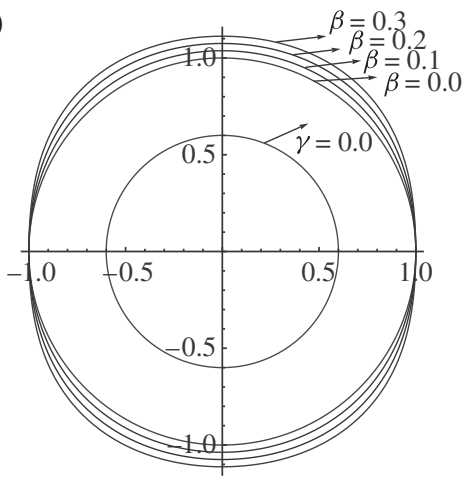

(b)

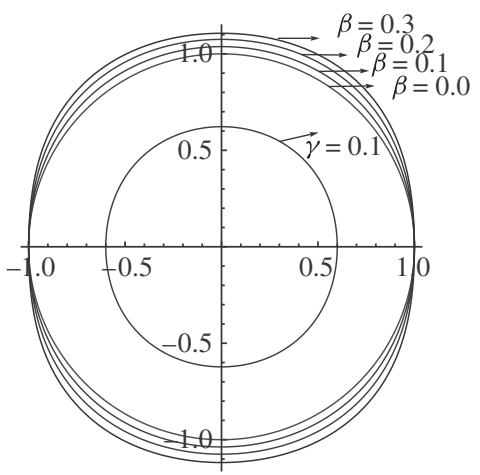

FIGURE 2. The shape of the shell with varying deformation parameter of the outer shell $\left(\beta=\beta_{2}=\beta_{4}\right)$. The deformation parameter of the inner shell $\left(\gamma=\gamma_{2}=\gamma_{4}\right)$ is fixed, and the separation parameter $\eta=0.6$.

Using (4.1) and carrying out the integration, we find

$$
D=4 \pi \mu U a\left[B_{2}+\frac{1}{5}\left(\Delta_{1} \beta_{2}+\Delta_{2} \gamma_{2}\right)+\frac{2}{35}\left(\Delta_{3} \beta_{4}+\Delta_{4} \gamma_{4}\right)\right]
$$

where $B_{2}, \Delta_{1}, \Delta_{2}, \Delta_{3}$ and $\Delta_{4}$ are constants whose expressions are lengthy and therefore not presented here.

It is interesting to note that although the boundary surface is given by the equations

$$
r=a\left[1+\sum_{m=2}^{\infty} \beta_{m} \vartheta_{m}(\zeta)\right] \text { and } r=b\left[1+\sum_{m=2}^{\infty} \gamma_{m} \vartheta_{m}(\zeta)\right]
$$

only the coefficients $\beta_{2}, \beta_{4}, \gamma_{2}$ and $\gamma_{4}$ contribute to the drag. This implies that the drag on the porous approximately spherical shell is relatively insensitive to the details of the surface geometry. This is similar to the observation made by Srinivasacharya [18].

If $\beta_{m}=0$ and $\gamma_{m}=0$ for $m>2$, the approximately spherical shell reduces to a spherical shell and the drag is

$$
D=4 \pi \mu U a B_{2} .
$$

The drag on a porous sphere $(\eta=0)$ with continuity of tangential stress $(\sigma=0)$ is

$$
D=\frac{12 \pi \mu U a \alpha^{2}(-\alpha \cosh (\alpha)+\sinh (\alpha))}{\alpha\left(3+2 \alpha^{2}\right) \cosh (\alpha)-3 \sinh (\alpha)},
$$

which agrees with the porous sphere case derived by Brinkman [4], Neale et al. [12] and Qin and Kaloni [16].

The changes of shape from porous spherical shell to porous approximately spherical shell are shown in Figures 2 and 3 . The variation of the drag coefficient $D_{\mathrm{N}}=$ $D /(4 \pi \mu U a)$ with permeability $k$ for fixed values of the outer deformation parameter $\beta=\beta_{2}=\beta_{4}$ and inner deformation parameter $\gamma=\gamma_{2}=\gamma_{4}$ with continuity of the stress $(\sigma=0)$ is shown in Figures 4 and 5. Figure 4(a) shows the variation of $D_{\mathrm{N}}$ with $k$ for 
(a)

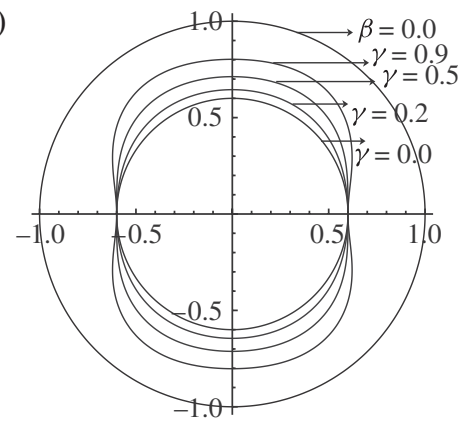

(b)

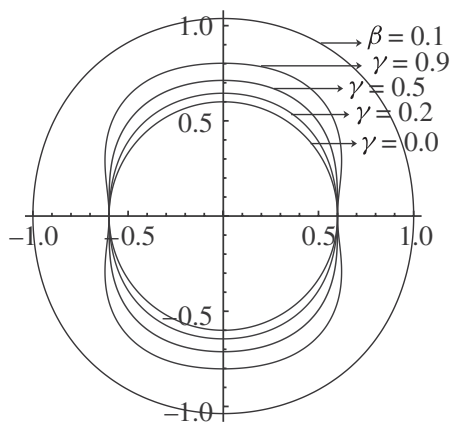

FIGURE 3. The shape of the shell with varying deformation parameter of the inner shell $\left(\gamma=\gamma_{2}=\gamma_{4}\right)$. The deformation parameter of the outer shell $\left(\beta=\beta_{2}=\beta_{4}\right)$ is fixed, and the separation parameter $\eta=0.6$.
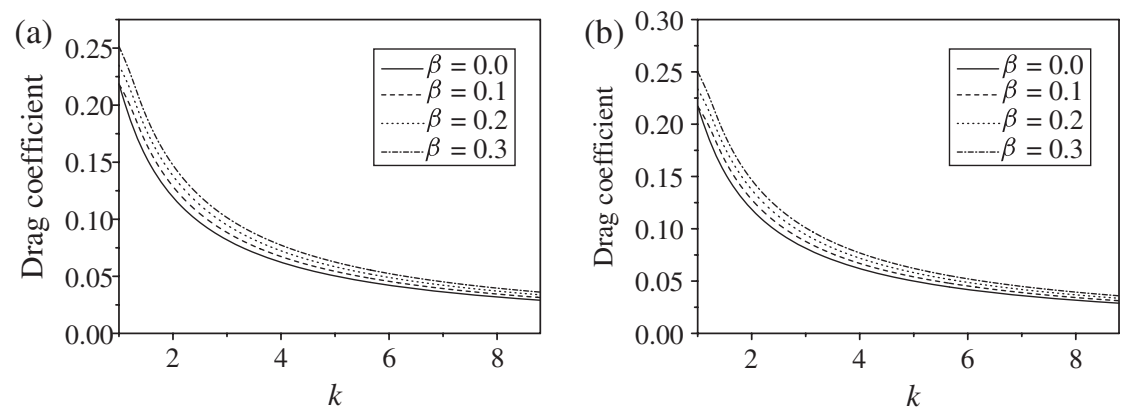

FIGURE 4. Variation of the drag coefficient with permeability $k$, with varying deformation parameter of the outer shell $\beta$. The stress jump coefficient $\sigma=0$, the separation parameter $\eta=0.6$ and the deformation parameter of the inner shell is fixed as (a) $\gamma=0.0$ and (b) $\gamma=0.1$.

fixed values of $\beta$ when the inner sphere is unperturbed $(\gamma=0)$. It is observed that $D_{\mathrm{N}}$ decreases as $k$ increases, and that $D_{\mathrm{N}}$ increases as $\beta$ increases. It is interesting to note that the drag coefficient on the spherical shell is less than that on the approximately spherical shell. Figure 4(b) shows the variation of $D_{\mathrm{N}}$ with $k$ for fixed values of $\beta$ when the inner sphere is perturbed with $\gamma=0.1$. There is an increase in $D_{\mathrm{N}}$ as $\beta$ increases. Figure 5(a) shows the variation of $D_{\mathrm{N}}$ versus $k$ with various values of $\gamma$ when the outer sphere is not deformed $(\beta=0)$. As $\gamma$ increases, there is a slight decrease in $D_{\mathrm{N}}$. Similar behaviour is observed in Figure 5(b) when the outer sphere is perturbed with $\beta=0.1$.

The effect of the stress jump coefficient $\sigma$ and permeability $k$ on the drag coefficient $D_{\mathrm{N}}$ at the porous-liquid interface is plotted in Figure 6. It is observed that $D_{\mathrm{N}}$ decreases as $\sigma$ increases. Moreover, $D_{\mathrm{N}}$ decreases as $k$ increases when there is a jump in the stress at the boundary. For positive values of $\sigma$, there is a reversal in the behaviour of the drag at a particular value of the permeability, the so-called critical permeability, $k_{\mathrm{c}}$ [3]. Beyond this value, $D_{\mathrm{N}}$ becomes negative, which is not 



FIGURE 5. Variation of the drag coefficient with permeability $k$, with varying deformation parameter of the inner shell $\gamma$. The stress jump coefficient $\sigma=0$, the separation parameter $\eta=0.6$ and the deformation parameter of the outer shell is fixed as (a) $\beta=0.0$ and (b) $\beta=0.1$.
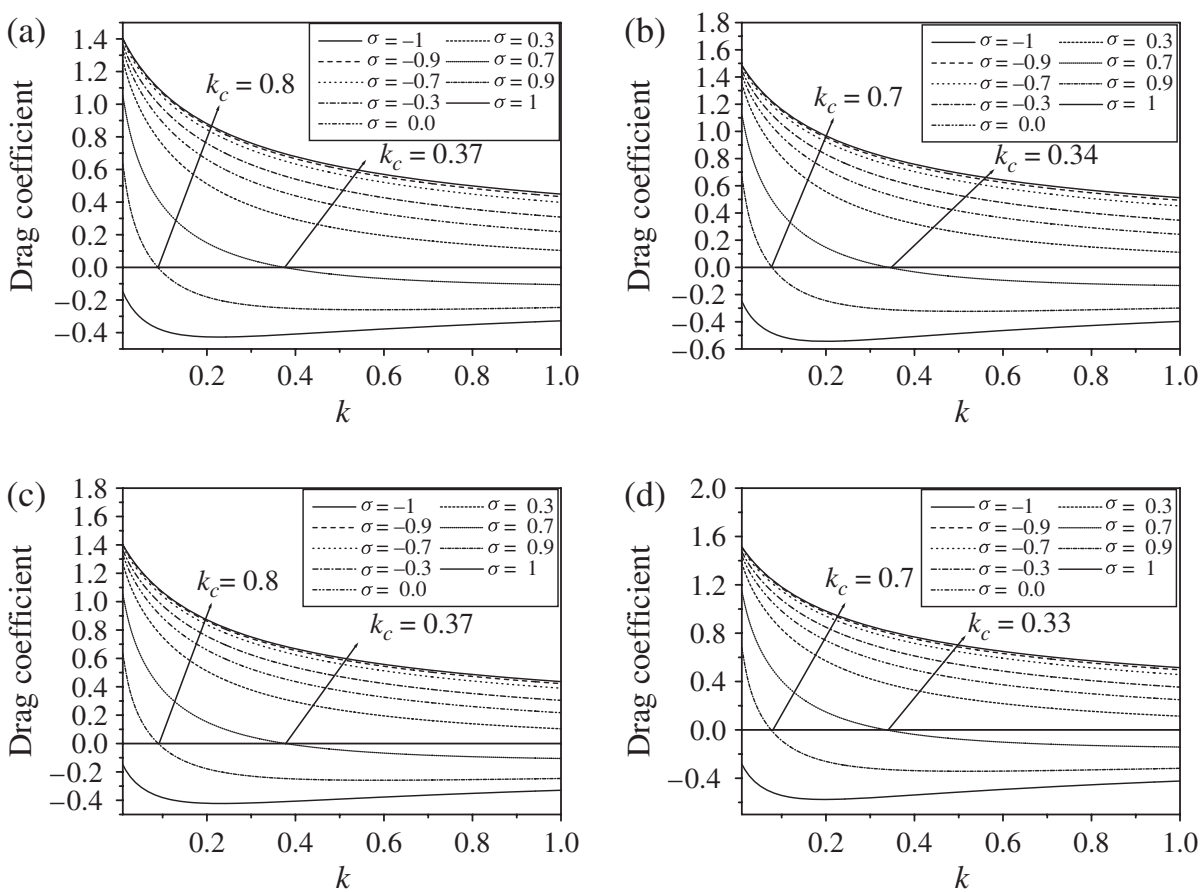

FIGURE 6. Variation of the drag coefficient with permeability $k$, with varying stress jump coefficient $\sigma$. The separation parameter $\eta=0.6$ and the deformation parameters of the outer shell $(\beta)$ and inner shell $(\gamma)$ are fixed as (a) $\beta=0.0, \gamma=0.0$, (b) $\beta=0.15, \gamma=0.0$, (c) $\beta=0.0, \gamma=0.1$ and (d) $\beta=0.2$, $\gamma=0.15$. 

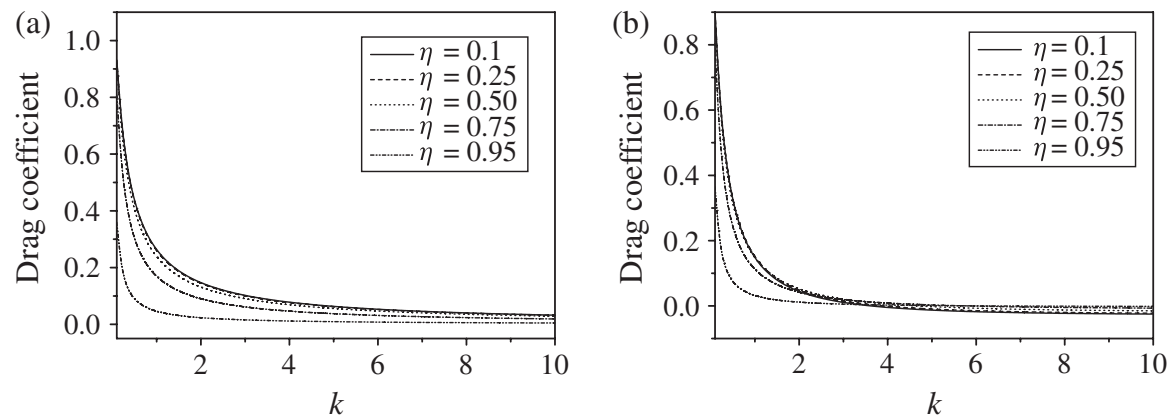

FIGURE 7. Variation of the drag coefficient with permeability $k$, with varying separation parameter $\eta$. The stress jump coefficient $\sigma$ and the deformation parameters of the outer shell $(\beta)$ and inner shell $(\gamma)$ are fixed as (a) $\beta=0.0, \gamma=0.0, \sigma=0$ and (b) $\beta=0.2, \gamma=0.15, \sigma=0.25$.

physically possible. Therefore, positive values of $\sigma$ cannot be considered beyond $k_{\mathrm{c}}$. The value of $k_{\mathrm{c}}$ decreases as the outer sphere deforms; there is not much difference in $k_{\mathrm{c}}$ as the inner sphere deforms. If negative values of $\sigma$ are considered in the stress jump condition (3.3), the shear stress of the external free flow region becomes greater than that of the porous region, generating a significant drag force on the porous surface for any value of the permeability $k$. But, for positive $\sigma$, although the shear stress of the external region becomes low, for a particular range of $k$ a significant drag force arises on the surface. Figure 7 shows the variation of $D_{\mathrm{N}}$ with $k$ for the case of a porous spherical shell with $\sigma=0$ and a porous approximately spherical shell with stress jump $(\sigma \neq 0)$. In both cases, $D_{\mathrm{N}}$ decreases with increasing $k$, and also with increasing separation parameter $\eta$. The critical permeability $k_{\mathrm{c}}$ increases as $\eta$ increases.

\section{Conclusion}

An exact solution for the problem of the creeping flow of an incompressible viscous liquid past a porous approximately spherical shell has been obtained by considering Brinkman's model in the porous region and Stokes' equations in the liquid region. At the porous-liquid interface, Ochoa-Tapia and Whitaker's [13, 14] stress jump boundary condition, continuity of the normal velocity and continuity of the pressure have been used. An expression for the drag on the porous approximately spherical shell has been obtained. It has been observed that the drag coefficient on the spherical shell is less than that on the approximately spherical shell. The drag decreases as the permeability increases, and also decreases as the stress jump coefficient $\sigma$ increases. For negative values of $\sigma$, the drag is always positive for any value of the permeability. However, for positive values of $\sigma$, the drag is positive only for a particular range of the permeability. Hence, we conclude that the stress jump condition, characterized by the stress jump coefficient $\sigma$, has a significant impact on the drag force. 


\section{References}

[1] G. S. Beavers and D. D. Joseph, "Boundary conditions at a naturally permeable wall", J. Fluid Mech. 30 (1967) 197-207; doi:10.1017/S0022112067001375.

[2] B. S. Bhatt and N. C. Sacheti, "Flow past a porous spherical shell using the Brinkman model", J. Phys. D: Appl. Phys. 27 (1994) 37-41; doi:10.1088/0022-3727/27/1/006.

[3] A. Bhattacharyya and G. P. Raja Sekhar, "Stokes flow inside a porous spherical shell: stress jump boundary condition”, Z. Angew. Math. Phys. 56 (2005) 475-496; doi:10.1007/s00033-004-2115-2.

[4] H. C. Brinkman, "A calculation of the viscous force exerted by a flowing fluid on a dense swarm of particles”, Appl. Sci. Res. 1 (1957) 27-34; doi:10.1007/BF02120313.

[5] P. Debye and A. M. Bueche, "Intrinsic viscosity, diffusion, and sedimentation rate of polymers in solution”, J. Chem. Phys. 16 (1948) 573-579; doi:10.1063/1.1746948.

[6] H. Happel and J. Brenner, Low Reynolds number hydrodynamics (Prentice Hall, Englewood Cliffs, NJ, 1965).

[7] I. P. Jones, "Low Reynolds number flow past a porous spherical shell", Proc. Cambridge Philos. Soc. 73 (1973) 231-238; doi:10.1017/S0305004100047642.

[8] D. D. Joseph and L. N. Tao, "The effect of permeability on the slow motion of a porous sphere in a viscous liquid", Z. Angew. Math. Mech. 44 (1964) 361-364; doi:10.1002/zamm.19640440804.

[9] A. V. Kuznetsov, "Analytical investigation of the fluid flow in the interface region between a porous medium and a clear fluid in channels partially filled with a porous medium", Appl. Sci. Res. 56 (1996) 53-67; doi:10.1007/BF02282922.

[10] A. V. Kuznetsov, "Analytical investigation of Couette flow in a composite channel partially filled with a porous medium and partially with a clear fluid", Int. J. Heat Mass Transfer 41 (1998) 2556-2560; doi:10.1016/S0017-9310(97)00296-2.

[11] K. Matsumoto and A. Suganuma, "Settling velocity of a permeable model floc", Chem. Eng. Sci. 32 (1977) 445-447; doi:10.1016/0009-2509(77)85009-4.

[12] G. Neale, N. Epstein and W. Nader, "Creeping flow relative to permeable spheres", Chem. Eng. Sci. 28 (1973) 1865-1874; doi:10.1016/0009-2509(73)85070-5.

[13] J. A. Ochoa-Tapia and S. Whitaker, "Momentum transfer at the boundary between a porous medium and a homogeneous fluid - I. Theoretical development", Int. J. Heat Mass Transfer 38 (1995) 2635-2646; doi:10.1016/0017-9310(94)00346-W.

[14] J. A. Ochoa-Tapia and S. Whitaker, "Momentum transfer at the boundary between a porous medium and a homogeneous fluid - II. Comparison with experiment", Int. J. Heat Mass Transfer 38 (1995) 2647-2655; doi:10.1016/0017-9310(94)00347-X.

[15] G. Ooms, P. E. Mijnlieff and H. L. Beckers, "Frictional force exerted by a flowing fluid on a permeable particle, with particular reference to polymer coils", J. Chem. Phys. 53 (1970) 4123-4130; doi:10.1063/1.1673911.

[16] Y. Qin and P. N. Kaloni, "A Cartesian-tensor solution of the Brinkman equation", J. Eng. Math. 22 (1988) 177-188; doi:10.1007/BF02383599.

[17] P. G. Saffman, "On the boundary condition at the surface of a porous medium", Stud. Appl. Math. 50 (1971) 93-101.

[18] D. Srinivasacharya, "Flow past a porous approximate spherical shell", Z. Angew. Math. Phys. 58 (2007) 646-658; doi:10.1007/s00033-006-6003-9.

[19] T. Zlatanovski, "Axisymmetric creeping flow past a porous prolate spheroidal particle using the Brinkman model”, Q. J. Mech. Appl. Math. 52 (1999) 111-126; doi:10.1093/qjmam/52.1.111. 